\title{
Role of etelcalcetide in the management of
} secondary hyperparathyroidism in hemodialysis patients: a review on current data and place in therapy

This article was published in the following Dove Press journal:

Drug Design, Development and Therapy

\author{
Claudia Friedl' \\ Emanuel Zitt ${ }^{2}$ \\ 'Department of Internal Medicine, \\ Clinical Division of Nephrology, \\ Medical University of Graz, Graz, \\ ${ }^{2}$ Department of Internal Medicine III, \\ Nephrology and Dialysis, Feldkirch \\ Academic Teaching Hospital, \\ Feldkirch, Austria
}

\begin{abstract}
Secondary hyperparathyroidism (sHPT) is a frequently occurring severe complication of advanced kidney disease. Its clinical consequences include extraskeletal vascular and valvular calcifications, changes in bone metabolism resulting in renal osteodystrophy, and an increased risk of cardiovascular morbidity and mortality. Calcimimetics are a cornerstone of parathyroid hormone (PTH)-lowering therapy, as confirmed by the recently updated 2017 Kidney Disease: Improving Global Outcomes chronic kidney disease - mineral and bone disorder clinical practice guidelines. Contrary to calcitriol or other vitamin D-receptor activators, calcimimetics reduce PTH without increasing serum-calcium, phosphorus, or FGF23 levels. Etelcalcetide is a new second-generation calcimimetic that has been approved for the treatment of sHPT in adult hemodialysis patients. Whereas the first-generation calcimimetic cinacalcet is taken orally once daily, etelcalcetide is given intravenously thrice weekly at the end of the hemodialysis session. Apart from improving drug adherence, etelcalcetide has proven to be more effective in lowering PTH when compared to cinacalcet, with an acceptable and comparable safety profile. The hope for better gastrointestinal tolerance with intravenous administration did not come true, as etelcalcetide did not significantly mitigate the adverse gastrointestinal effects associated with cinacalcet. Enhanced adherence and strong reductions in PTH, phosphorus, and FGF23 could set the stage for a future large randomized controlled trial to demonstrate that improved biochemical control of mineral metabolism with etelcalcetide in hemodialysis patients translates into cardiovascular and survival benefits and better health-related quality of life.
\end{abstract}

Keywords: calcimimetic, chronic kidney disease, dialysis, etelcalcetide, secondary hyperparathyroidism

\section{Introduction}

Secondary hyperparathyroidism (sHPT) is common in hemodialysis patients. This complication of chronic kidney disease (CKD) is caused by an attempt to control the disturbed calcium, phosphorus, and vitamin D metabolism. SHPT causes vascular and soft-tissue calcification and leads to disturbances of mineral metabolism. Combining these mineral, bone, and cardiovascular abnormalities, the clinical syndrome is now known as CKD-related mineral and bone disorder (CKD-MBD).

Clinically, sHPT causes vascular and valvular calcification and changes in bone metabolism that lead to renal osteodystrophy. ${ }^{1}$ Furthermore, in large international observational studies, an independent association between increasing parathyroid hormone (PTH) and cardiovascular and all-cause mortality was found, especially
Correspondence: Emanuel Zitt Department of Internal Medicine III, Nephrology and Dialysis, Feldkirch Academic Teaching Hospital, 47 Carinagasse, Feldkirch 6800, Austria Tel +4355223032700

Fax +435522 3037506 Email emanuel.zitt@lkhf.at 
for PTH $>600 \mathrm{pg} / \mathrm{mL} .^{2,3}$ sHPT-associated high FGF23 is independently associated with left ventricular hypertrophy, ${ }^{4}$ cardiovascular events, ${ }^{5}$ and premature death. ${ }^{6}$ CKD-MBD abnormalities have also been implicated as risk factors for the very rare but devastating calcific and thrombotic arteriolopathy calciphylaxis ${ }^{7}$ and lead to reduced health-related quality of life (HRQoL). The indication for sHPT treatment results from these clinical consequences.

In this article, we briefly summarize the pathogenesis of sHPT in CKD, with emphasis on the key molecular regulators that are targeted by calcimimetics, briefly touch on sHPT treatment options with regard to the updated Kidney Disease: Improving Global Outcomes (KDIGO) CKD-MBD practice guidelines, review the lessons learnt from the oral first-generation calcimimetic cinacalcet with a focus on drug adherence, and finally describe preclinical and clinical data of the new intravenous second-generation calcimimetic etelcalcetide.

\section{Pathogenesis of secondary hyperparathyroidism in chronic kidney disease}

sHPT generally develops in stage $3 \mathrm{CKD}$ with an estimated glomerular filtration rate $<60 \mathrm{~mL} / \mathrm{min} / 1.73 \mathrm{~m}^{2}$. Almost all hemodialysis patients suffer from sHPT ${ }^{8,9}$ It is characterized by normal or slightly decreased serum-calcium levels, initial normophosphatemia followed by hyperphosphatemia, low $1,25(\mathrm{OH})_{2} \mathrm{D}_{3}$ (calcitriol) concentration, increasing levels of FGF23, a decrease in plasma-soluble Klotho and the development of renal osteodystrophy. ${ }^{9,10}$ These changes cause parathyroid-cell hyperplasia accompanied by increased synthesis and secretion of PTH. ${ }^{11}$ Reduced renal phosphorusexcretion capacity leads to transient increases in serum phosphorus and concomitantly decreased ionized calcium concentrations. To counteract these alterations, increased PTH corrects these changes in mineral metabolism as it reduces the tubular reabsorption of phosphorus and increases the reabsorption of calcium. ${ }^{12}$ Additionally, the phosphatonin FGF23 decreases serum-phosphorus levels, due to reduced tubular phosphorus reabsorption independent of PTH. For full activity and activation of its receptors FGFR1 and FGFR3, it requires the presence of its coreceptor Klotho. ${ }^{13}$ Contrary to increasing FGF23 levels, tubular Klotho expression and levels of soluble Klotho decrease with declining kidney function. ${ }^{14}$ PTH itself stimulates FGF23 secretion directly and indirectly through enhanced synthesis of calcitriol secondary to the PTH-induced stimulation of tubular $1 \alpha$-hydroxylase. ${ }^{15-17}$ On the other hand, FGF23 lowers the secretion of PTH, although decreased Klotho and FGFR1 expression on hyperplastic parathyroid cells attenuate this effect. ${ }^{18,19}$

The key molecular regulators of parathyroid function and PTH secretion are the cell-surface calcium-sensing receptor (CaSR) and nuclear vitamin D receptor (VDR). A reduction in blood ionized calcium concentration rapidly stimulates the secretion of preformed PTH, prolonged hypocalcemia increases PTH synthesis, and this causes parathyroid-cell hyperplasia. ${ }^{20}$ Activation of the VDR lowers PTH transcription, whereas decreased calcitriol stimulates PTH synthesis. $^{21}$

Elevated phosphorus reduces the activity of tubular $1 \alpha$-hydroxylase and lowers the synthesis of calcitriol. ${ }^{22}$ Also, it stimulates PTH production independently of changes in calcium and calcitriol concentration ${ }^{23}$ and directly increases parathyroid-cell proliferation, ${ }^{24}$ due to downregulation of parathyroid CaSR and VDR. ${ }^{25}$ Low serum concentrations of calcium and phosphorus and high PTH stimulate $1 \alpha$-hydroxylase and increase calcitriol production, whereas FGF23 and calcitriol itself reduce enzyme activity. ${ }^{26}$ With progressive sHPT, parathyroid-cell hyperplasia is characterized by reduced expression of CaSR, VDR, and FGFR1. 18,19,27,28 This parathyroid resistance promotes and aggravates the development of severe sHPT.

\section{sHPT treatment: 2017 KDIGO CKD-MBD guidelines}

Current treatment options for sHPT include: reducing oral phosphorus uptake by dietary phosphorus restriction, especially limiting phosphate additives in processed foods and favoring plant-based protein sources, and by the use of phosphate binders; the inhibition of PTH synthesis and secretion by the supplementation of calcitriol or other VDR activators (VDRAs) or the use of calcimimetics; finally, surgical parathyroidectomy is a valuable option in refractory cases after pharmacotherapy has failed. Unchanged, the recently updated KDIGO CKD-MBD clinical practice guidelines suggest for chronic hemodialysis patients maintenance of PTH levels in the range of approximately two to nine times the upper limit of normal for the particular PTH assay in use. In these patients, calcimimetics, VDRAs, or a combination of calcimimetics with VDRAs can be used to achieve this recommended target range. ${ }^{29} \mathrm{With}$ an evidence level of $2 \mathrm{~B}$, no single approach is preferred over another. Until now, there have been no prospective randomized controlled trials (RCTs) of phosphate binders or VDRAs indicating a benefit on patient-level outcomes except improvements in bone disease with alfacalcidol. ${ }^{30}$ Contrary to VDRAs, which tend 
to increase serum-calcium, phosphorus, and FGF23 levels, while decreasing PTH, the first-generation calcimimetic cinacalcet effectively reduces PTH without increasing calcium, phosphorous, or FGF23. ${ }^{31,32}$ Treatment with cinacalcet improves biochemical parameters of sHPT, with significantly more patients achieving recommended target levels compared to patients treated with a standard regimen containing calcitriol or another VDRA. ${ }^{33,34}$ Even in patients with persistent or recurrent HPT after parathyroidectomy, cinacalcet is effective. ${ }^{35}$ Experimental data has found calcitriol and paricalcitol induce soft-tissue and aortic calcification in uremic rats, while cinacalcet monotherapy did not cause extraskeletal calcifications and attenuated the deleterious effect when given in combination with these VDRAs. ${ }^{36}$

\section{Lessons learnt from the first- generation calcimimetic cinacalcet}

Cinacalcet is the first calcimimetic drug approved for the treatment of sHPT in adult dialysis patients. It has been commercially available in the US since 2004, in Europe since 2005, and in Japan since 2008. As an allosteric modulator, cinacalcet increases the sensitivity of the CaSR to extracellular ionized calcium, leading to decreased PTH synthesis and secretion. ${ }^{37}$ It is taken orally once daily, with a recommended starting dose of $30 \mathrm{mg}$. To achieve PTH targets, uptitration to a maximum of single-dose $180 \mathrm{mg}$ is possible. ${ }^{31}$

In prevalent hemodialysis patients with moderate-severe SHPT and preexisting vascular or valvular calcification, treatment with cinacalcet in combination with low-dose VDRAs attenuated the progression of coronary artery and aortic valve calcification over 52 weeks compared to a treatment regimen based on flexible doses of a VDRA alone when assessed by the calcium-volume score, but slightly missed statistical significance with the Agatston score $(P=0.07)$ in the ADVANCE trial. ${ }^{38}$ In a post hoc analysis of protocoladherent patients in the same study, significantly attenuated progression of cardiovascular calcification was found, even using the Agatston score. ${ }^{39}$ In the large randomized placebocontrolled, double-blind EVOLVE trial conducted in 3,883 dialysis patients with SHPT, patients treated with cinacalcet on top of standard care showed better control of SHPT and lower risk of developing severe unremitting HPT compared to the placebo group. ${ }^{40}$ However, the unadjusted primary composite end point (time to death or first occurrence of a nonfatal cardiovascular event, including myocardial infarction, hospitalization for unstable angina, heart failure, and peripheral vascular event) showed a nonsignificant reduction in intention-to-treat analysis with cinacalcet. Adjusted for imbalances in baseline characteristics or nonadherence, a nominally significant reduction in the primary composite end point was found. ${ }^{41}$ Additionally, further prespecified secondary analyses of EVOLVE demonstrated a significant risk reduction for parathyroidectomy ${ }^{40}$ or the development of calciphylaxis ${ }^{42}$ with the use of cinacalcet.

With regard to bone turnover and histology, cinacalcet has been shown to decrease histomorphometric markers of bone turnover after 6-12 months of treatment in 77 dialysis patients with biopsy-proven high bone turnover. It generally improved bone histology, with most of the patients presenting with mild hyperparathyroid bone disease or mixed uremic osteodystrophy, and significantly increased the proportion of patients with normal bone histology (from 0 patients at baseline to 20 patients after 12 months). ${ }^{43}$

No definite answer can be given to the question of whether cinacalcet has an impact on patient-reported outcome HRQoL. In a combined analysis of data from three similarly designed Phase III RCTs enrolling a total of 1,136 patients (665 cinacalcet, 471 controls), HRQoL improved slightly for the Medical Outcomes Study Short Form 36 physical component-summary score and the specific domains of bodily pain and general health perception. ${ }^{44}$ A systematic review about the effect of cinacalcet on QoL in patients with endstage KD (ESKD) and sHPT, including two observational studies and one EVOLVE-based RCT, found no significant change from baseline in HRQoL with cinacalcet treatment. ${ }^{45}$ HRQoL is influenced by many factors, and it remains difficult to assess the true and sole benefit of one single intervention. Moreover, most RCTs exploring patient-reported HRQoL as a secondary end point are not adequately powered to detect small or modest differences in this outcome.

\section{Drug adherence with oral cinacalcet}

Despite improved control of sHPT using cinacalcet in combination with or without other treatment options for SHPT, one demanding challenge is poor adherence to cinacalcet therapy, which can impair long-term sHPT control. In the literature, nonadherence to cinacalcet varies: $45.6 \%-71 \%$. ${ }^{46-48}$ Gincherman et al investigated the refill-based adherence rate for cinacalcet in 79 hemodialysis patients, and found a 1 -year medication-possession rate $>80 \%$ (indicating consistent medication use) of $29 \%$ for cinacalcet. ${ }^{46}$ The authors hypothesized that the lower adherence rate with cinacalcet had been the result of the high incidence of gastrointestinal side effects. The most frequent side effects of cinacalcet are gastrointestinal, primarily nausea and vomiting. In an RCT by Block et al, the frequency of nausea was $32 \%$ in patients 
treated with cinacalcet versus $19 \%$ in the placebo group $(P<0.001) .{ }^{31}$ These results were in line with those obtained from an RCT conducted by Lindberg et al (nausea 30\% vs $22 \%) .{ }^{49}$ In both trials, vomiting was more common in the cinacalcet arm compared to placebo (30\% vs $16 \%$ and $23 \%$ vs $12 \%$, respectively). ${ }^{31,49}$ In observational studies of daily clinical practice, the reported frequency of gastrointestinal side effects with cinacalcet was lower than in RCTs. In the observational ECHO trial conducted in 1,865 dialysis patients receiving cinacalcet, nausea was experienced by $5 \%$ and vomiting by $3 \%$ of the study cohort. ${ }^{50}$ The rate of treatment discontinuation due to these gastrointestinal side effects was $<5 \%$ in the study by Block et $\mathrm{al}^{31}$ and $3 \%$ in the ECHO trial. ${ }^{50}$ Therefore, the high nonadherence to cinacalcet could not be explained only by its side effects.

Economic factors may also contribute to poor cinacalcet adherence. In a retrospective study, Park et al analyzed the data of more than 11,700 Medicare beneficiaries on dialysis in the USA. Only $35 \%$ of these patients were adherent to cinacalcet (medication-possession rate $\geq 80 \%$ ). Additionally, they observed differences between dialysis-specific medications and non-dialysis-specific medications, with higher adherence and persistence to non-dialysis-specific medications. These differences in adherence may be attributable to different medication costs by therapeutic classes. The costs of dialysis medications were significantly higher compared to non-dialysis-specific medications. ${ }^{48}$

A further possible explanation for high nonadherence to cinacalcet could be the high oral drug load in dialysis patients. Chiu et al found a median daily pill burden of 19 in chronic dialysis patients, and a quarter of them were prescribed more than 25 pills per day. Drugs for the treatment of sHPT accounted for about half the daily pill burden. ${ }^{51}$ Since the consequences of nonadherence to medications in the treatment of sHPT are generally not immediately noticed by the patient, one could speculate that this may be a further reason for lower adherence to cinacalcet compared to other medication. Importantly, higher adherence to cinacalcet was associated with inpatient savings of US\$4,000-\$8,900/patient/ year in a retrospective study on 4,923 dialysis patients. ${ }^{47}$ Therefore, better adherence may not only influence patients' health but has also high economic impact.

Given the fact that poor long-term adherence to prescribed medication is a common problem in dialysis patients ${ }^{52,53}$ and associated with higher morbidity and mortality, as well as increased treatment costs, ${ }^{54}$ strategies to improve adherence in dialysis patients are of particular importance. One strategy to decrease nonadherence could be the intravenous application of drugs during or after hemodialysis. Such an approach has become possible with etelcalcetide. The hope to avoid gastrointestinal side-effects and improve drug adherence with intravenous administration during hemodialysis have been drivers in the development of the secondgeneration calcimimetic etelcalcetide, which is described in detail in the following sections.

\section{Mode of action and preclinical data of etelcalcetide}

Etelcalcetide (Parsabiv; Amgen, Thousand Oaks, CA, USA), formerly known as AMG416 or velcalcetide, is a new second-generation calcimimetic that was approved for the treatment of sHPT in adult hemodialysis patients in the EU in November 2016, in Japan in December 2016, and in the US in February 2017. Etelcalcetide is a small peptide containing eight amino acids with a molecular weight of 1,048 Da. It causes long-lasting allosteric activation of the CaSR through the formation of a covalent disulfide bond between the D-cysteine in etelcalcetide and cysteine 482 in the extracellular domain of the CaSR. ${ }^{55}$

Similarly to cinacalcet, etelcalcetide causes rapid and dose-dependent decrease of PTH (maximal reduction within 2 hours in healthy subjects; after approximately 30 minutes in hemodialysis patients), calcium, phosphorus, and FGF23 levels. But in contrast to cinacalcet, etelcalcetide can activate the CaSR even under calcium-free conditions, indicating its additional function as a direct $\mathrm{CaSR}$ agonist. However, approximately 30 times as much ligand is required to generate the same magnitude of response as observed in the presence of calcium. The pharmacokinetic profile of etelcalcetide in patients with CKD differs from cinacalcet. Etelcalcetide is almost exclusively cleared by the kidney through glomerular filtration. Therefore, its plasma-elimination half-life significantly increases with declining renal function, with a short effective half-life of 3-5 days in patients with ESKD. A single intravenous dose can lower PTH levels for up to 72 hours in patients on hemodialysis. This longer half-life allows intravenous administration thrice weekly at the end of each hemodialysis session. After administration of a single intravenous dose, regular 4-hour hemodialysis sessions three times a week are responsible for around $60 \%$ of its clearance, whereas $3 \%$ are eliminated in urine, $6 \%$ in feces, and $31 \%$ by nonspecific mechanisms. Etelcalcetide is resistant to enzymatic degradation by proteases and does not interact with cytochrome P450. It undergoes biotransformation by disulfide exchange with endogenous thiols, resulting in the reversible formation of albumin-peptide conjugates. 
Table I Differences between first- and second-generation calcimimetics

\begin{tabular}{|c|c|c|}
\hline & Cinacalcet & Etelcalcetide \\
\hline Class & $\begin{array}{l}\text { First-generation } \\
\text { calcimimetic type II } \\
\text { Small organic molecule }\end{array}$ & $\begin{array}{l}\text { Second-generation } \\
\text { calcimimetic type II } \\
\text { Octapeptide }\end{array}$ \\
\hline $\begin{array}{l}\text { Molecular formula } \\
\text { Molecular weight }\end{array}$ & $\begin{array}{l}\mathrm{C}_{22} \mathrm{H}_{23} \mathrm{~F}_{3} \mathrm{~N} \\
394 \mathrm{Da}\end{array}$ & $\begin{array}{l}\mathrm{C}_{38} \mathrm{H}_{73} \mathrm{~N}_{21} \mathrm{O}_{10} \mathrm{~S}_{2} \\
\mathrm{I}, 048 \mathrm{Da}\end{array}$ \\
\hline $\begin{array}{l}\text { Mode of action at } \\
\text { CaSR }\end{array}$ & Allosteric modulator & $\begin{array}{l}\text { Allosteric modulator } \\
\text { and direct agonist }\end{array}$ \\
\hline $\begin{array}{l}\text { Location of } \\
\text { interaction with CaSR }\end{array}$ & Transmembrane domain & Extracellular domain \\
\hline $\begin{array}{l}\text { Mode of } \\
\text { administration }\end{array}$ & Daily oral & $\begin{array}{l}\text { Thrice-weekly } \\
\text { intravenously at the } \\
\text { end of hemodialysis } \\
\text { session }\end{array}$ \\
\hline
\end{tabular}

Abbreviation: CaSR, calcium-sensing receptor.

These conjugates are not dialyzable, because of their molecular weight of $67 \mathrm{kDa}$. In the presence of L-cysteine, reverse disulfide exchange reforms etelcalcetide, the forward reaction forming the conjugate being faster than the reverse reaction. ${ }^{56}$ Table 1 provides a short overview of the key differences between first- and second-generation calcimimetics.

In addition to its biochemical effects on mineral and bone metabolism, etelcalcetide has also been shown to reduce parathyroid-cell proliferation and increase expression of the CaSR, VDR, and FGFR1 in parathyroid cells in a rodent uremic model. ${ }^{57}$ Despite a similar PTH-lowering effect, etelcalcetide treatment significantly lowered aortic calcium content and prevented medial aortic calcification in uremic rats with SHPT, whereas paricalcitol did not show these beneficial effects. ${ }^{58} \mathrm{~A}$ direct effect on vascular endothelial ${ }^{59}$ or smooth-muscle cells ${ }^{60}$ expressing the CaSR or an FGF23-dependent pathway could be responsible for these effects. Furthermore, animal experimental data have provided the first evidence that etelcalcetide might be beneficial in the management of renal osteodystrophy. In nephrectomized rats with established sHPT, etelcalcetide attenuated SHPT-associated increase in cortical bone porosity, mineralization defects, and bone-marrow fibrosis and improved bone strength. ${ }^{61}$

\section{Clinical data}

The efficacy and safety of etelcalcetide in SHPT treatment in dialysis patients have been investigated in several RCTs. ${ }^{62-66}$ In a single-dose crossover Phase II trial by Martin et al,${ }^{66}$ the efficacy of etelcalcetide was investigated in 28 hemodialysis patients suffering from sHPT. Patients were enrolled in one of five cohorts and received either a single intravenous dose of etelcalcetide $(5,10,20,40,60 \mathrm{mg})$ or placebo. Treatment with etelcalcetide was well tolerated and resulted in dosedependent decreases in PTH levels. In addition, administration of etelcalcetide at doses $\geq 10 \mathrm{mg}$ was associated with dose-dependent reductions in FGF23 and serum-calcium concentrations and resulted in diminished interdialytic increase in serum phosphorus compared to placebo.

In 2015 , Bell et a ${ }^{62}$ reported on results of a multicenter, double-blind, randomized, placebo-controlled, doseescalating trial. This Phase II study included 78 hemodialysis patients with baseline PTH levels $\geq 350 \mathrm{pg} / \mathrm{mL}$. Subjects were divided into three cohorts: patients in cohort 1 received either $5 \mathrm{mg}$ etelcalcetide or placebo thrice weekly after each hemodialysis session for 2 weeks, and those in cohorts 2 and 3 were treated with $10 \mathrm{mg}$ or $5 \mathrm{mg}$ etelcalcetide or placebo at the end of each dialysis for 4 weeks. The primary end point for cohorts 2 and 3 was defined as mean percentage change in PTH levels from baseline. After 4 weeks, PTH had decreased significantly by $49.4 \%$ with $10 \mathrm{mg}$ of etelcalcetide and by $33.0 \%$ with $5 \mathrm{mg}$. The proportion of patients with $\geq 30 \%$ PTH reduction was $76.2 \%$ in etelcalcetide-treated patients vs $9.5 \%$ in the placebo group $(P<0.0001)$. Treatment with etelcalcetide was also associated with a decrease in serum-calcium and FGF23 levels. Approximately 40\% of study participants reported at least one treatment-emergent adverse event (TEAE), but the incidence of TEAEs was not dose-dependent and no patient discontinued the study due to a TEAE.

Block et al ${ }^{64}$ conducted two parallel, Phase III, doubleblind, RCTs of 1,023 dialysis patients from six nations with moderate-severe sHPT (PTH $>400 \mathrm{pg} / \mathrm{mL}$ ). Patients received either etelcalcetide or placebo after each hemodialysis session for 26 weeks in addition to conventional sHPT therapy. The primary end point was the proportion of patients attaining $>30 \%$ PTH reduction from baseline during the efficacy-assessment phase from week 20 to 27 . The starting dose of etelcalcetide was $5 \mathrm{mg}$, and treatment was adjusted according to PTH and calcium levels to a maximum dose of $15 \mathrm{mg}$. In the first trial, 508 patients were enrolled, of whom 254 received etelcalcetide (median per-session dose $7.1 \mathrm{mg}$ ). The second trial included 515 patients, with 255 randomized to the study drug (median per-session dose $5.0 \mathrm{mg}$ ). In both trials, patients receiving etelcalcetide were significantly more likely to achieve the primary efficacy end point: $74.0 \%$ vs $8.3 \%(P<0.001)$ and $75.3 \%$ vs $9.6 \%(P<0.001)$, respectively. In addition, more patients in the etelcalcetide group achieved a mean PTH level $\leq 300 \mathrm{pg} / \mathrm{mL}$ (49.6\% vs $5.1 \%$ and $53.3 \%$ vs $4.6 \%, P<0.001)$. Compared to placebo, 
serum-calcium levels decreased significantly in the etelcalcetide group, but the proportion of patients receiving calcium supplements, calcium-containing phosphate binders, or VDRAs increased. Dialysate-calcium concentrations were found to be higher in etelcalcetide-treated patients. The calcium-lowering effect of the calcimimetic was observed early, with the lowest calcium concentrations found during treatment weeks 10-12. Furthermore, etelcalcetide decreased serum-phosphorus and FGF23 levels. In terms of adverse events, diarrhea (10.7\% vs $8.6 \%$ ), nausea (10.7\% vs $6.2 \%$ ), vomiting ( $8.9 \%$ vs $5.1 \%$ ), and symptomatic reduction in corrected serum calcium $<8.3 \mathrm{mg} / \mathrm{dL}(7.0 \%$ vs $0.2 \%)$ were more common in patients with etelcalcetide.

The results of a further Phase III trial investigating the efficacy and safety of etelcalcetide were recently reported by Fukagawa et al. ${ }^{65}$ This multicenter, randomized, doubleblind, placebo-controlled, parallel-group study was conducted in 155 Japanese hemodialysis patients with PTH levels $\geq 300 \mathrm{pg} / \mathrm{mL}$. Patients randomized to the study drug received etelcalcetide thrice weekly after each hemodialysis. The starting dose was $5 \mathrm{mg}$, and according to PTH and calcium levels, the dose was subsequently adjusted to single doses of $2.5-15 \mathrm{mg}$ at 4 -week intervals for 12 weeks. The mean dose of etelcalcetide was $7.8 \mathrm{mg}$ at the end of the study. The primary end point was the proportion of patients with PTH levels of 60-240 pg/mL on day 85, the PTH target range recommended by the Japanese Society for Dialysis Therapy. Compared to placebo, patients randomized to etelcalcetide more often achieved this primary end point $(59.0 \% \mathrm{vs}$ $1.3 \%$ ). With etelcalcetide, a higher proportion of patients achieved $\geq 30 \%$ reduction in PTH from baseline (76.9\% vs $5.2 \%$ ). In addition, treatment with etelcalcetide was associated with a decrease in serum calcium, phosphorus and FGF23. Drug-related AEs were reported in 19.2\% of patients receiving etelcalcetide versus $3.9 \%$ in the placebo group.

In a head-to-head Phase III study, Block et al ${ }^{63}$ investigated the efficacy and safety of etelcalcetide versus cinacalcet in 683 hemodialysis patients with moderate-severe sHPT (PTH $\geq 500 \mathrm{pg} / \mathrm{mL}$ ). Patients randomized to etelcalcetide $(\mathrm{n}=340)$ received the drug thrice weekly intravenously at the end of each hemodialysis session and oral placebo daily for a total duration of 26 weeks. Patients in the control group received daily oral cinacalcet and thrice-weekly intravenous placebo after hemodialysis. The starting dose of etelcalcetide was $5 \mathrm{mg}$, and cinacalcet was administered with an initial dose of $30 \mathrm{mg}$. Dose titrations were performed every 4 weeks during the first 4 months (etelcalcetide $2.5-5 \mathrm{mg}$ titration, final dose range $2.5-15 \mathrm{mg}$; cinacalcet $30 \mathrm{mg}$ titration, final dose range $30-180 \mathrm{mg}$ ) with a centrally measured target PTH level of
$100-300 \mathrm{pg} / \mathrm{mL}$. The primary end point of this randomized, double-blind, double-dummy trial was noninferiority of etelcalcetide at achieving $>30 \%$ PTH reduction from baseline during the efficacy-assessment phase (weeks 20-27). Key secondary end points included superiority in attaining biochemical end points ( $>50 \%$ and $>30 \%$ reduction in $\mathrm{PTH})$ and mean number of weekly days with self-reported nausea and vomiting over the first 8 weeks. The median weekly dose of etelcalcetide was $15.0 \mathrm{mg}$ and the median daily cinacalcet dose $51.4 \mathrm{mg}$. Etelcalcetide was noninferior to cinacalcet in reducing PTH levels, and demonstrated superiority for several end points. The proportion of patients achieving $>30 \%$ reduction in PTH was $68.2 \%$ in the etelcalcetide group versus $57.7 \%$ in the cinacalcet group $(P=0.004)$. Significantly more patients in the etelcalcetide group achieved $>50 \%$ reduction in $\mathrm{PTH}$ levels (52.4\% vs $40.2 \%, P=0.001)$. Furthermore, the proportion of patients with $>30 \%$ reduction in FGF23 was higher in the etelcalcetide-treated group ( $74.4 \%$ vs $57.5 \%, P<0.0001)$. Compared with cinacalcet, treatment with etelcalcetide was also associated with greater reduction in serum-calcium and phosphorus levels. The use of calcium supplements, calciumcontaining phosphate binders, and VDRAs, as well as the proportion of patients treated with a higher dialysate-calcium concentration, increased in both groups. No significant difference was observed in self-reported nausea and vomiting between the etelcalcetide- and cinacalcet-treated group. Therefore, these gastrointestinal side effects seem to be a systemic effect rather than a local class effect of calcimimetics, maybe mediated by an activation of the CaSR in nonparathyroid target organs. The overall safety and tolerability were similar between the two groups (Table 2). As predicted by Phase II trials, application of etelcalcetide was frequently accompanied by reduced serum calcium concentrations (based

Table 2 Incidence of adverse events in head-to-head comparison between cinacalcet and etelcalcetide

\begin{tabular}{|c|c|c|}
\hline & $\begin{array}{l}\text { Cinacalcet } \\
(n=34 I)\end{array}$ & $\begin{array}{l}\text { Etelcalcetide } \\
(n=338)\end{array}$ \\
\hline & n (\%) & n (\%) \\
\hline Calcium reduction ${ }^{\mathrm{a}}$ & $204(59.8)$ & $233(68.9)$ \\
\hline Hypocalcemia $^{\mathrm{b}}$ & $8(2.3)$ & I7 (5.0) \\
\hline Muscle spasms & $20(5.9)$ & $22(6.5)$ \\
\hline Pain in extremity & $14(4.1)$ & $17(5.0)$ \\
\hline Paresthesia & $6(1.8)$ & $7(2.1)$ \\
\hline Nausea & $77(22.6)$ & $62(18.3)$ \\
\hline Vomiting & 47 (I3.8) & $45(13.3)$ \\
\hline Diarrhea & $35(10.3)$ & $21(6.2)$ \\
\hline Heart failure & $2(0.6)$ & $10(3.0)$ \\
\hline Death & $6(1.8)$ & $9(2.7)$ \\
\hline
\end{tabular}

Notes: ${ }^{a}$ Defined as reduction in serum albumin-corrected calcium $<8.3 \mathrm{mg} / \mathrm{dL}$ that resulted in a medical intervention; 'defined as symptomatic reduction in serum albumin-corrected calcium $<8.3 \mathrm{mg} / \mathrm{dL}$. 


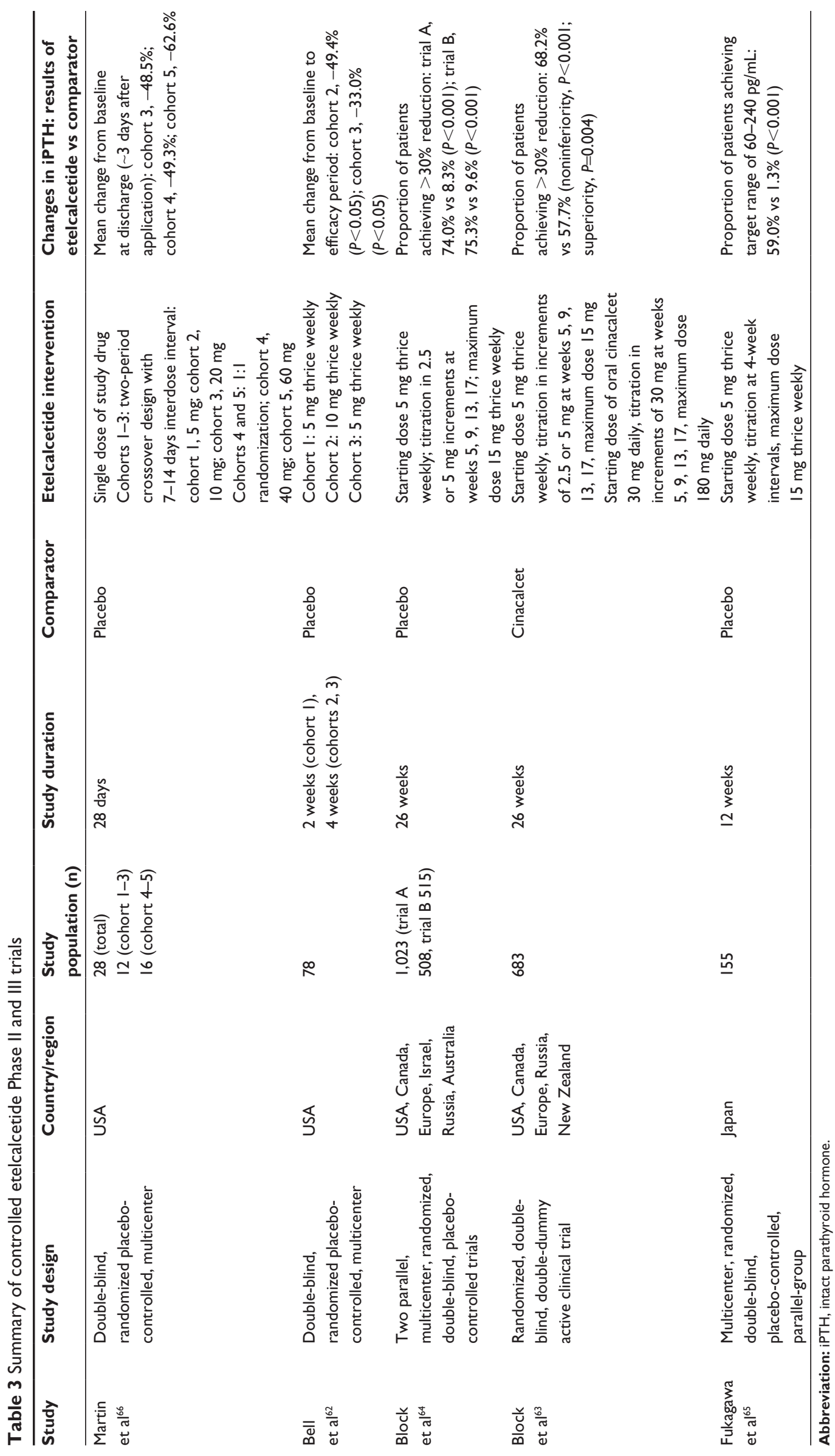


on albumin-corrected total calcium), reflecting asymptomatic hypocalcemia in Phase III trials. Not surprisingly, a reduction in serum calcium $<8.3 \mathrm{mg} / \mathrm{dL}$ was the most common AE. Overt symptomatic hypocalcemia was found in $6.7 \%-7.2 \%$ of etelcalcetide-treated patients in the placebo-controlled trials (compared to $0 \%-0.4 \%$ of placebo-treated patients) and in $5 \%$ of patients in the head-to-head comparison with cinacalcet $(2.3 \%)$. The clinical significance of calcimimetic-induced hypocalcemia remains unclear, as it is rarely associated with clinical sympoms. ${ }^{31,50}$ The 2017 KDIGO CKD-MBD practice guidelines no longer recommend the maintenance of serum calcium concentration within the normal reference range in dialysis patients; rather, it suggests avoiding hypercalcemia and tolerates mild and asymptomatic calcimimetic-associated hypocalcemia to avoid inappropriate calcium loading in these patients. ${ }^{29}$ This approach is supported by a very recent post hoc analysis of the EVOLVE trial, which found severe hypocalcemia (total serum calcium $<7.5 \mathrm{mg} / \mathrm{dL}$ ) within the first 16 weeks after the first administered dose in $18.4 \%$ of patients in the cinacalcet group versus $4.4 \%$ in the placebo group. ${ }^{67}$ This event was not dose-dependent, but associated with higher baseline PTH values, reflecting an increased likelihood of developing hypocalcemia with increasing sHPT severity. In the majority of patients, hypocalcemia resolved within 14 days without modification of sHPT treatment (reduction/ discontinuation of cinacalcet, initiation/increase of VDRAs, initiation of calcium-containing phosphate binder). Further studies are necessary to determine the effect of etelcalcetide on vascular and skeletal health considering the hypocalcemic effect and associated changes in VDRA therapy or calcium supplementation. Nevertheless, a baseline albumin-corrected serum calcium level $\geq 8.3 \mathrm{mg} / \mathrm{dL}$, in accordance with the Phase III trials, should be a prerequisite prior to the initiation of etelcalcetide in our opinion.

Cinacalcet should be discontinued for at least 7 days prior to the initiation of the intravenous calcimimetic in patients who switch to etelcalcetide. Table 3 briefly summarizes the key characteristics of published controlled Phase II and III trials of etelcalcetide.

To date, no controlled studies directly comparing etelcalcetide with placebo, cinacalcet, or surgical parathyroidectomy with regard to hard clinical end points, such as mortality, cardiovascular events, fractures and parathyroidectomy in patients with ESKD have been conducted. The same holds true for parathyroidectomy, which has never been compared to calcimimetics in an RCT. This renders cost-effectiveness analyses more difficult. With rising healthcare costs and limited resources, such analyses focusing on economic and clinical value of a specific treatment become more and more important.

Recently, a decision-analysis model was developed to assess the lifetime cost-effectiveness of etelcalcetide versus cinacalcet, excluding dialysis costs. ${ }^{68}$ In this model, the long-term efficacy of etelcalcetide was extrapolated from its effect on PTH reduction in three Phase III trials (versus placebo and cinacalcet ${ }^{63,64}$ and from clinical event-rate data from EVOLVE. ${ }^{41}$ Compared with cinacalcet, the incremental cost-effectiveness ratio (cost per quality-adjusted life year [QALY]gained) of etelcalcetide was $€ 1,355 / Q A L Y$ assuming the same weekly calcimimetic drug cost, €24,521/QALY assuming $15 \%$ higher weekly costs for etelcalcetide, and $€ 47,687 /$ QALY assuming 30\% higher weekly costs for etelcalcetide. Definite cost-effectiveness of etelcalcetide may vary from one country to another, dependent on countryspecific drug costs, clinical event costs, reimbursement policy, and the willingness-to-pay threshold.

\section{Conclusion}

The new second-generation calcimimetic etelcalcetide effectively reduces PTH, phosphorus, calcium, and FGF23 in hemodialysis patients with an acceptable safety profile. Intravenous administration at the end of a hemodialysis session promises better drug adherence, reduces pill burden, and might thus allow improved management of sHPT compared to previous standard care. Enhanced adherence and better control of sHPT could set the stage for a future large RCT to demonstrate that improved biochemical control of mineral metabolism with etelcalcetide translates into affordable cardiovascular and survival benefits and better QoL for hemodialysis patients.

\section{Disclosure}

EZ has received speakers' honoraria from Amgen. CF reports no conflicts of interest in this work.

\section{References}

1. Moe S, Drüeke T, Cunningham J, et al. Definition, evaluation, and classification of renal osteodystrophy: a position statement from Kidney Disease: Improving Global Outcomes (KDIGO). Kidney Int. 2006; 69(11):1945-1953.

2. Floege J, Kim J, Ireland E, et al. Serum iPTH, calcium and phosphate, and the risk of mortality in a European haemodialysis population. Nephrol Dial Transplant. 2011;26(6):1948-1955.

3. Tentori F, Wang M, Bieber BA, et al. Recent changes in therapeutic approaches and association with outcomes among patients with secondary hyperparathyroidism on chronic hemodialysis: the DOPPS study. Clin J Am Soc Nephrol. 2015;10(1):98-109.

4. Faul C, Amaral AP, Oskouei B, et al. FGF23 induces left ventricular hypertrophy. J Clin Invest. 2011;121(11):4393-4408. 
5. Scialla JJ, Xie H, Rahman M, et al. Fibroblast growth factor- 23 and cardiovascular events in CKD. J Am Soc Nephrol. 2014;25(2):349-360.

6. Gutierrez OM, Mannstadt M, Isakova T, et al. Fibroblast growth factor 23 and mortality among patients undergoing hemodialysis. $N$ Engl $J$ Med. 2008;359(6):584-592.

7. Zitt E, Konig M, Vychytil A, et al. Use of sodium thiosulphate in a multi-interventional setting for the treatment of calciphylaxis in dialysis patients. Nephrol Dial Transplant. 2013;28(5):1232-1240.

8. de Boer IH, Gorodetskaya I, Young B, Hsu CY, Chertow GM. The severity of secondary hyperparathyroidism in chronic renal insufficiency is GFR-dependent, race-dependent, and associated with cardiovascular disease. J Am Soc Nephrol. 2002;13(11):2762-2769.

9. Levin A, Bakris GL, Molitch M, et al. Prevalence of abnormal serum vitamin D, PTH, calcium, and phosphorus in patients with chronic kidney disease: results of the study to evaluate early kidney disease. Kidney Int. 2007;71(1):31-38.

10. Isakova T, Wahl P, Vargas GS, et al. Fibroblast growth factor 23 is elevated before parathyroid hormone and phosphate in chronic kidney disease. Kidney Int. 2011;79(12):1370-1378.

11. Cunningham J, Locatelli F, Rodriguez M. Secondary hyperparathyroidism: pathogenesis, disease progression, and therapeutic options. Clin J Am Soc Nephrol. 2011;6(4):913-921.

12. Slatopolsky E, Caglar S, Pennell JP, et al. On the pathogenesis of hyperparathyroidism in chronic experimental renal insufficiency in the dog. J Clin Invest. 1971;50(3):492-499.

13. Kurosu H, Kuro OM. The Klotho gene family as a regulator of endocrine fibroblast growth factors. Mol Cell Endocrinol. 2009;299(1):72-78.

14. Hu MC, Kuro-o M, Moe OW. The emerging role of Klotho in clinical nephrology. Nephrol Dial Transplant. 2012;27(7):2650-2657.

15. Fan Y, Bi R, Densmore MJ, et al. Parathyroid hormone 1 receptor is essential to induce FGF23 production and maintain systemic mineral ion homeostasis. FASEB J. 2016;30(1):428-440.

16. Lopez I, Rodriguez-Ortiz ME, Almaden Y, et al. Direct and indirect effects of parathyroid hormone on circulating levels of fibroblast growth factor 23 in vivo. Kidney Int. 2011;80(5):475-482.

17. Meir T, Durlacher K, Pan Z, et al. Parathyroid hormone activates the orphan nuclear receptor Nurr1 to induce FGF23 transcription. Kidney Int. 2014;86(6):1106-1115.

18. Canalejo R, Canalejo A, Martinez-Moreno JM, et al. FGF23 fails to inhibit uremic parathyroid glands. J Am Soc Nephrol. 2010;21(7): $1125-1135$.

19. Galitzer H, Ben-Dov IZ, Silver J, Naveh-Many T. Parathyroid cell resistance to fibroblast growth factor 23 in secondary hyperparathyroidism of chronic kidney disease. Kidney Int. 2010;77(3):211-218.

20. Silver J. Molecular mechanisms of secondary hyperparathyroidism. Nephrol Dial Transplant. 2000;15 Suppl 5:2-7.

21. Friedl C, Zitt E. Vitamin D prohormone in the treatment of secondary hyperparathyroidism in patients with chronic kidney disease. Int $J$ Nephrol Renovasc Dis. 2017;10:109-122.

22. Slatopolsky E, Brown A, Dusso A. Pathogenesis of secondary hyperparathyroidism. Kidney Int Suppl. 1999;73:S14-S19.

23. Almaden Y, Hernandez A, Torregrosa V, et al. High phosphate level directly stimulates parathyroid hormone secretion and synthesis by human parathyroid tissue in vitro. J Am Soc Nephrol. 1998;9(10): $1845-1852$.

24. Naveh-Many T, Rahamimov R, Livni N, Silver J. Parathyroid cell proliferation in normal and chronic renal failure rats: the effects of calcium, phosphate, and vitamin D. J Clin Invest. 1995;96(4):1786-1793.

25. Nakajima K, Umino K, Azuma Y, et al. Stimulating parathyroid cell proliferation and PTH release with phosphate in organ cultures obtained from patients with primary and secondary hyperparathyroidism for a prolonged period. J Bone Miner Metab. 2009;27(2):224-233.

26. Bikle D. Vitamin D: production, metabolism, and mechanisms of action. In: de Groot LJ, Chrousos G, Dungan K, et al, editors. Endotext. South Dartmouth (MA): MDText; 2000.

27. Gogusev J, Duchambon P, Hory B, et al. Depressed expression of calcium receptor in parathyroid gland tissue of patients with hyperparathyroidism. Kidney Int. 1997;51(1):328-336.
28. Fukuda N, Tanaka H, Tominaga Y, Fukagawa M, Kurokawa K, Seino Y. Decreased 1,25-dihydroxyvitamin $\mathrm{D}_{3}$ receptor density is associated with a more severe form of parathyroid hyperplasia in chronic uremic patients. J Clin Invest. 1993;92(3):1436-1443.

29. Ketteler M, Block GA, Evenepoel P, et al. Executive summary of the 2017 KDIGO chronic kidney disease-mineral and bone disorder (CKDMBD) guideline update: what's changed and why it matters. Kidney Int. 2017;92(1):26-36.

30. Hamdy NA, Kanis JA, Beneton MN, et al. Effect of alfacalcidol on natural course of renal bone disease in mild to moderate renal failure. BMJ. 1995;310(6976):358-363.

31. Block GA, Martin KJ, de Francisco AL, et al. Cinacalcet for secondary hyperparathyroidism in patients receiving hemodialysis. $N$ Engl $J$ Med. 2004;350(15):1516-1525.

32. Zitt E, Fouque D, Jacobson SH, et al. Serum phosphorus reduction in dialysis patients treated with cinacalcet for secondary hyperparathyroidism results mainly from parathyroid hormone reduction. Clin Kidney $J$. 2013;6(3):287-294.

33. Li D, Shao L, Zhou H, Jiang W, Zhang W, Xu Y. The efficacy of cinacalcet combined with conventional therapy on bone and mineral metabolism in dialysis patients with secondary hyperparathyroidism: a meta-analysis. Endocrine. 2013;43(1):68-77.

34. Zhang Q, Li M, You L, et al. Effects and safety of calcimimetics in end stage renal disease patients with secondary hyperparathyroidism: a meta-analysis. PLoS One. 2012;7(10):e48070.

35. Zitt E, Rix M, Torres PU, et al. Effectiveness of cinacalcet in patients with recurrent/persistent secondary hyperparathyroidism following parathyroidectomy: results of the ECHO study. Nephrol Dial Transplant. 2011;26(6):1956-1961.

36. Lopez I, Mendoza FJ, Aguilera-Tejero E, et al. The effect of calcitriol, paricalcitol, and a calcimimetic on extraosseous calcifications in uremic rats. Kidney Int. 2008;73(3):300-307.

37. Nemeth EF, Heaton WH, Miller M, et al. Pharmacodynamics of the type II calcimimetic compound cinacalcet HCl.J Pharmacol Exp Ther. 2004;308(2):627-635.

38. Raggi P, Chertow GM, Torres PU, et al. The ADVANCE study: a randomized study to evaluate the effects of cinacalcet plus low-dose vitamin D on vascular calcification in patients on hemodialysis. Nephrol Dial Transplant. 2011;26(4):1327-1339.

39. Ureña-Torres PA, Floege J, Hawley CM, et al. Protocol adherence and the progression of cardiovascular calcification in the ADVANCE study. Nephrol Dial Transplant. 2013;28(1):146-152.

40. Parfrey PS, Chertow GM, Block GA, et al. The clinical course of treated hyperparathyroidism among patients receiving hemodialysis and the effect of cinacalcet: the EVOLVE trial. $J$ Clin Endocrinol Metab. 2013;98(12):4834-4844.

41. EVOLVE Trial Investigators. Effect of cinacalcet on cardiovascular disease in patients undergoing dialysis. $N$ Engl J Med. 2012;367(26): 2482-2494.

42. Floege J, Kubo Y, Floege A, Chertow GM, Parfrey PS. The effect of cinacalcet on calcific uremic arteriolopathy events in patients receiving hemodialysis: the EVOLVE trial. Clin J Am Soc Nephrol. 2015;10(5): $800-807$.

43. Behets GJ, Spasovski G, Sterling LR, et al. Bone histomorphometry before and after long-term treatment with cinacalcet in dialysis patients with secondary hyperparathyroidism. Kidney Int. 2015;87(4): 846-856.

44. Cunningham J, Danese M, Olson K, Klassen P, Chertow GM. Effects of the calcimimetic cinacalcet $\mathrm{HCl}$ on cardiovascular disease, fracture, and health-related quality of life in secondary hyperparathyroidism. Kidney Int. 2005;68(4):1793-1800.

45. van der Plas WY, Dulfer RR, Engelsman AF, et al. Effect of parathyroidectomy and cinacalcet on quality of life in patients with end-stage renal disease-related hyperparathyroidism: a systematic review. Nephrol Dial Transplant. 2017;32(11):1902-1908.

46. Gincherman Y, Moloney K, McKee C, Coyne DW. Assessment of adherence to cinacalcet by prescription refill rates in hemodialysis patients. Hemodial Int. 2010;14(1):68-72. 
47. Lee A, Song X, Khan I, et al. Association of cinacalcet adherence and costs in patients on dialysis. J Med Econ. 2011;14(6):798-804.

48. Park H, Rascati KL, Lawson KA, Barner JC, Richards KM, Malone DC. Adherence and persistence to prescribed medication therapy among Medicare part $\mathrm{D}$ beneficiaries on dialysis: comparisons of benefit type and benefit phase. J Manag Care Spec Pharm. 2014;20(8):862-876.

49. Lindberg JS, Culleton B, Wong G, et al. Cinacalcet $\mathrm{HCl}$, an oral calcimimetic agent for the treatment of secondary hyperparathyroidism in hemodialysis and peritoneal dialysis: a randomized, double-blind, multicenter study. J Am Soc Nephrol. 2005;16(3):800-807.

50. Ureña $\mathrm{P}$, Jacobson SH, Zitt E, et al. Cinacalcet and achievement of the NKF/K-DOQI recommended target values for bone and mineral metabolism in real-world clinical practice: the ECHO observational study. Nephrol Dial Transplant. 2009;24(9):2852-2859.

51. Chiu YW, Teitelbaum I, Misra M, de Leon EM, Adzize T, Mehrotra R. Pill burden, adherence, hyperphosphatemia, and quality of life in maintenance dialysis patients. Clin J Am Soc Nephrol. 2009;4(6): 1089-1096.

52. Burnier M, Pruijm M, Wuerzner G, Santschi V. Drug adherence in chronic kidney diseases and dialysis. Nephrol Dial Transplant. 2015; 30(1):39-44.

53. Ghimire S, Castelino RL, Lioufas NM, Peterson GM, Zaidi ST. Nonadherence to medication therapy in haemodialysis patients: a systematic review. PLoS One. 2015;10(12): 0144119.

54. Loghman-Adham M. Medication noncompliance in patients with chronic disease: issues in dialysis and renal transplantation. Am J Manag Care. 2003;9(2):155-171.

55. Alexander ST, Hunter T, Walter S, et al. Critical cysteine residues in both the calcium-sensing receptor and the allosteric activator AMG 416 underlie the mechanism of action. Mol Pharmacol. 2015;88(5): $853-865$.

56. Wu B, Melhem M, Subramanian R, et al. Clinical pharmacokinetics and pharmacodynamics of etelcalcetide, a novel calcimimetic for treatment of secondary hyperparathyroidism in patients with chronic kidney disease on hemodialysis. J Clin Pharmacol. Epub 2018 Mar 13.

57. Walter S, Baruch A, Dong J, et al. Pharmacology of AMG 416 (velcalcetide), a novel peptide agonist of the calcium-sensing receptor, for the treatment of secondary hyperparathyroidism in hemodialysis patients. J Pharmacol Exp Ther. 2013;346(2):229-240.

58. Yu L, Tomlinson JE, Alexander ST, et al. Etelcalcetide, a novel calcimimetic, prevents vascular calcification in a rat model of renal insufficiency with secondary hyperparathyroidism. Calcif Tissue Int. 2017; 101(6):641-653.
59. Ziegelstein RC, Xiong Y, He C, Hu Q. Expression of a functional extracellular calcium-sensing receptor in human aortic endothelial cells. Biochem Biophys Res Commun. 2006;342(1):153-163.

60. Smajilovic S, Hansen JL, Christoffersen TE, et al. Extracellular calcium sensing in rat aortic vascular smooth muscle cells. Biochem Biophys Res Commun. 2006;348(4):1215-1223.

61. Li X, Yu L, Asuncion F, et al. Etelcalcetide (AMG 416), a peptide agonist of the calcium-sensing receptor, preserved cortical bone structure and bone strength in subtotal nephrectomized rats with established secondary hyperparathyroidism. Bone. 2017;105:163-172.

62. Bell G, Huang S, Martin KJ, Block GA. A randomized, double-blind, phase 2 study evaluating the safety and efficacy of AMG 416 for the treatment of secondary hyperparathyroidism in hemodialysis patients. Curr Med Res Opin. 2015;31(5):943-952.

63. Block GA, Bushinsky DA, Cheng S, et al. Effect of etelcalcetide vs cinacalcet on serum parathyroid hormone in patients receiving hemodialysis with secondary hyperparathyroidism: a randomized clinical trial. JAMA. 2017;317(2):156-164.

64. Block GA, Bushinsky DA, Cunningham J, et al. Effect of etelcalcetide vs placebo on serum parathyroid hormone in patients receiving hemodialysis with secondary hyperparathyroidism: two randomized clinical trials. JAMA. 2017;317(2):146-155.

65. Fukagawa M, Yokoyama K, Shigematsu T, et al. A phase 3, multicentre, randomized, double-blind, placebo-controlled, parallel-group study to evaluate the efficacy and safety of etelcalcetide (ONO-5163/AMG 416), a novel intravenous calcimimetic, for secondary hyperparathyroidism in Japanese haemodialysis patients. Nephrol Dial Transplant. 2017;32(10):1723-1730.

66. Martin KJ, Pickthorn K, Huang S, et al. AMG 416 (velcalcetide) is a novel peptide for the treatment of secondary hyperparathyroidism in a single-dose study in hemodialysis patients. Kidney Int. 2014;85(1): 191-197.

67. Floege J, Tsirtsonis K, Iles J, Drüeke TB, Chertow GM, Parfrey P. Incidence, predictors and therapeutic consequences of hypocalcemia in patients treated with cinacalcet in the EVOLVE trial. Kidney Int. Epub 2018 Mar 7

68. Stollenwerk B, Iannazzo S, Akehurst R, et al. A decision-analytic model to assess the cost-effectiveness of etelcalcetide vs. cinacalcet. Pharmacoeconomics. 2018;36(5):603-612.
Drug Design, Development and Therapy

\section{Publish your work in this journal}

Drug Design, Development and Therapy is an international, peerreviewed open-access journal that spans the spectrum of drug design and development through to clinical applications. Clinical outcomes, patient safety, and programs for the development and effective, safe, and sustained use of medicines are the features of the journal, which

\section{Dovepress}

has also been accepted for indexing on PubMed Central. The manuscript management system is completely online and includes a very quick and fair peer-review system, which is all easy to use. Visit http://www.dovepress.com/testimonials.php to read real quotes from published authors. 